\title{
LA FORMACIÓN DEL CARÁCTER EN EL PENSAMIENTO DE ELENA G. DE WHITE: UNA PERSPECTIVA DE EDUCACIÓN INTEGRAL
}

\author{
Victoria Martinez Tejada ${ }^{1 \mathrm{a}}$ \\ Universidad Peruana Unión ${ }^{1}$ \\ Orcid ID: https://orcid.org/0000-0001-8474-4784 ${ }^{1}$
}

Recibido: 01 de julio de 2018

Aceptado: 10 de enero 2019

\begin{abstract}
Resumen
El estudio del carácter es un tema vigente para los educadores, porque a partir de ello se construyen los lineamientos curriculares de los diversos programas de formación educativa que en todos sus niveles buscan la educación integral. A fin de tener una dirección clara en cuanto al carácter y su formación, se hizo necesario desarrollar una investigación de enfoque cualitativo, con un diseño de investigación yuxtapuesto de teoría fundamentada emergente y narrativa, para conocer el pensamiento de Elena G. De White con respecto a este tópico a lo largo de sus escritos (1844 - 1915).
\end{abstract}

Palabras claves: Carácter, lineamientos curriculares, educación integral, Elena G. de White.

\section{THE FORMATION OF CHARACTER IN THE WRITINGS OF ELLEN G. WHITE: A PERSPECTIVE OF HOLISTIC EDUCATION}

\begin{abstract}
The study of character is a subject that is relevant for Adventist educators, because from now on it is the subject in which the curriculum guidelines of the various educational training programs are developed and constructed at the levels of formation. In order to have a clear direction regarding the character and its scope and implications, it is important to develop a qualitative approach research study, with a juxtaposed design of emergent and narrative based theory, to know the thoughts of Ellen G. White with regard to this topic and its evolution throughout her prophetic ministry (1844 - 1915). The Seventh-day Adventist Church (SDA) considers that Ellen G. White is a prophet of God and her writings are considered as authoritative for the diverse guidelines of the work of the church and its parishioners.
\end{abstract}

Keywords: Character, curricular framework, holistic education, Ellen G. White.

\footnotetext{
a Correspondencia al autor

E-mail: jehita@gmail.com
} 


\section{Introducción}

El estudio del carácter es un desafío para todo sistema educativo. La educación actual demanda la necesidad de atender el aspecto del ser en su totalidad. Por otro lado, los actuales sistemas educativos solo evidencian la transmisión de buenos conocimientos, y muestran la existencia de un desbalance que deja de lado aspectos importantes para el desarrollo de vida del educando, pues más allá de demandar el perfeccionamiento académico, el estudiante necesita ser mejor persona.

La condición humana que permite la distinción en nuestra existencia es aquella que se ve reflejada por un carácter que sostiene la vida toda. Para los grupos religiosos es sabido que la condición espiritual permea toda dimensión de la vida, y muestran otros elementos que añaden valor en las relaciones humanas, llevando a la persona a una realidad de autocuidado en todo aspecto: académico, social, mental y de conciencia del cuidado del medio ambiente; vale decir, evidenciar un desarrollo integral, armonioso, donde se puede ver simetría en cada faceta de la vida y demostrando que todo conocimiento recibido es importante al perseguir este fin.

El objetivo de esta investigación es observar con precisión los generadores que permiten la formación de un carácter según los escritos de Elena G. De White y que permiten entender la complejidad de la esencia del término carácter, como éste se construye y evidencia en toda persona. En base al estudio, es importante encontrar las fuentes primarias de los escritos de Elena G. De White, la cual es considerada como una de las fundadoras del sistema educativo adventista.

\section{Breve revisión}

Esta investigación presenta como objetivo principal el encontrar la información sobre el tema del desarrollo del carácter en los escritos primarios de Elena G. De White, tomando aquellos que tienen una relación precisa con el tema. Se encontraron un total de 34 citas las directamente relacionadas al constructo de desarrollo del carácter.

Fowler (1977) hizo una revisión de los escritos de Elena G. De White con respecto al desarrollo del carácter y concluyó que, para el pensamiento de White, el carácter es el centro y está constituido por todos los aspectos de la vida del ser humano. El carácter es necesario para la obra redentora y educativa. Los factores personales y relacionales como el concepto de sí mismo, la herencia, el medio ambiente, la salud, la inteligencia, los hábitos dietéticos, la vocación intencional, el estudio de la Biblia, la meditación, la 
oración, la adoración, las relaciones entre compañeros y el servicio altruista influyen en el desarrollo del carácter.

Grajales (2005), considera que el ambiente o atmósfera escolar es la expresión más tangible del currículo institucional, y esto contiene lo que experimentan los estudiantes y las demás personas de la escuela, con respeto al trato. Este ambiente escolar puede contribuir a la formación de carácter cristiano.

Zavala (2006) señala que la formación del carácter, desde la perspectiva de Elena G. De White se contempla como el proceso en el cual los hábitos de la mente, el corazón y la conducta interviene durante toda la vida para dar como fruto el carácter.

\section{Metodología}

Se usó un enfoque cualitativo, un diseño de investigación yuxtapuesta de teoría fundamentada emergente y narrativo, y un tipo denominado de tópico y de codificación abierta como refiere Hernández, Fernández y Baptista (2010) y Mertens (2005).

Se revisaron 1600 citas y por el año de publicación se fueron depurando; fueron codificadas y analizadas, y se generó un modelo: a) Se seleccionaron aquellas que se aproximaban al concepto y la tipificación del constructo carácter, b) Se analizaron y codificaron las citas, c) Las citas generaron subcategorías las que a su vez, nos permitieron sistematizarlas en categorías, d) fueron ilustradas por diagramas, d) Se contextualizó cada cita en el marco histórico en que fueron escritas, para comprender la razón de ser de cada una de ellas, e) finalmente se discutieron y aproximaron las categorías al silabo, documento curricular cercano al estudiante, a una entrevista y a la observación de clase.

\section{Análisis de la investigación}

Para el logro de hallazgos en la presente investigación se siguieron los siguientes pasos:

- En la ventana buscadora se ubicó el tópico en español: "carácter es..."

- Esta búsqueda se hizo a través del EGW Índex que se ejecuta desde la página web del White State (https://egwwritings.org/?searchform=topicalIndex).

- Buscando en todas las citas que contenían la palabra carácter como sustantivo, y no como adjetivo, que es la forma como muchas veces aparece, al ser encontrado como sustantivo; se toma la cita, para así encontrar aquellos temas que lo componen, de los cuales posteriormente fluye la investigación de la formación del 
carácter en una perspectiva de educación integral, en el pensamiento de Elena G. De White.

- En un principio se abordaron citas de libros escritos por Elena G. De White, traducidos al español, pues era una materia prima que se consideraba seria oportuna tomar.

- Posteriormente en la ventana buscadora de la misma fuente del White State, Se ubicó el tópico en inglés: "Character is", solo en fuentes en inglés.

- Luego se buscó las citas que hablarán de la formación del carácter desde las fuentes primarias de los escritos de Elena G. De White, en el idioma original, el inglés, quiere decir, aquellas aparecidas entre los años 1844 y 1915.

- Compréndase como fuente primaria, todo escrito que fue realizado mientras la escritora estaba en vida, a través de cartas y manuscritos, a partir de ellas es que se elaboran los diferentes libros, seleccionando estos de acuerdo a las diferentes temáticas que aborda cada libro.

- Algunas citas también aparecían ya constituidas en los libros escritos en inglés; desde allí se pesquisó hacia el testimonio de donde procedió, sea en la editorial de la época, Review and Herald, alguna carta o manuscrito.

- Se revisaron las citas desde los testimonios, basados en manuscritos y cartas. Aceptando que los escritos de Elena G. D White, efectuados en vida, son registrados con la más alta confiabilidad de los mismos, pues se contaba con su presencia en el año en que fueron escritas, siendo ella la directa revisora y también 
la responsable de la edición de los mismos, pues además de autora, era en aquel entonces, una fideicomisaria.

- Se sistematizaron las citas por año. Partiendo del de mayor antigüedad, en los años en vida de Elena G. De White hasta las citas más cercanas al año 1915, que fue el año en el que murió.

- Se hizo un análisis comparativo interpretativo en el cual aparecen las fuentes primarias en inglés traducidas al español y también un análisis de aquellas citas encontradas en español que tienen su origen en fuentes primarias.

- Se contextualizó cada cita en el contexto histórico del año en que fue escrita, a través de una línea de tiempo.

- Se ejecutó un doublé review con las citas que se tradujeron del inglés al español, esto se hizo a través del centro de idiomas de la Universidad Peruana Unión, con su directora.

- Se ubicaron las repeticiones de publicación y por afinidad y relación se agruparon, generando así las subcategorías, las cuales, al ser sistematizadas, se constituyeron en categorías respectivamente.

- Se consolidan las categorías y subcategorías que conforman el carácter, con palabras no siempre literales de los escritos de Elena G. De White, pero que engloban los temas encontrados en ellos.

Se confirmó la presencia de algunas fuentes primarias corroborando lo hallado en el $E G W$ Índex que se ejecuta desde la página web del White State (https://egwwritings.org/?searchform=topicalIndex) con los escritos, conservados en físico en el Centro de Investigaciones White - Perú, el cual forma parte del White State - USA, confirmando así la fidelidad de los escritos primarios. Esto permitió la categorización del concepto carácter, Al revisar las primeras fuentes, en las primeras etapas de esta investigación, básicamente las fuentes fueron los libros publicados en español de Elena G. De White. Al sistematizar estas los primeros hallazgos fueron 4 las categorías formadoras del carácter: Manejo de los pensamientos, servicio a los demás, dependencia de Dios y la interacción social.

Luego de estudiar las fuentes en su idioma original, el inglés, una vez avanzada la investigación, las categorías encontradas en las fuentes primarias finalmente quedaron en 6: Estudio de la Palabra de Dios, manejo de los pensamientos, relación con Dios, servicio a los demás, interacción social y cuidado del cuerpo. Todas ellas engloban otras 
temáticas denominadas subcategorías; por ello insistimos que en su redacción no se precisa igualdad de palabras necesariamente, aunque en alguna de ellas si aparecen, presentado tener igualdad de escritura con las sub categorías encontradas en las citas.

Finalmente se organizaron las categorías y sub categorías en la formación del carácter.

\section{Resultados}

\section{Cuadro 1}

Categorías, sub categorías y fuentes primarias

\begin{tabular}{|c|c|c|c|}
\hline $\mathrm{N}$ & $\begin{array}{c}\text { Categorías } \\
\text { formadoras del } \\
\text { carácter }\end{array}$ & Sub categoría & $\begin{array}{c}\text { Fuente primaria } \\
\text { (Escritos de Elena G. de White) }\end{array}$ \\
\hline \multirow{8}{*}{1} & \multirow{8}{*}{$\begin{array}{l}\text { Estudio de la } \\
\text { Biblia }\end{array}$} & \multirow{6}{*}{ Estudio de la Biblia } & The Review and Herald, June 12, 1888. \\
\hline & & & $\begin{array}{l}\text { White. E. G. (1911). The acts of the apostle. } \\
483 .\end{array}$ \\
\hline & & & Testimonies for the Church 4:376 (1881) \\
\hline & & & Testimonies for the Church 4:69 (1876). \\
\hline & & & El Ministerio de Curación, 364 (1905). \\
\hline & & & Carta 27, 1872. \\
\hline & & El Espíritu Santo por la Biblia & The Review and Herald, 25 de julio de 1899. \\
\hline & & La meditación & Testimonies for the Church. 5:113 (1882). \\
\hline \multirow{11}{*}{2} & \multirow{11}{*}{$\begin{array}{l}\text { El manejo de los } \\
\text { pensamientos }\end{array}$} & \multirow{2}{*}{ El manejo de los pensamientos } & The Review and Herald, June 12, 1888. \\
\hline & & & The Review and Herald, 3 de mayo de 1881 \\
\hline & & Ejercicio de la mente & $\begin{array}{l}\begin{array}{l}\text { Historia de los Patriarcas y Profetas, } 650 \\
(1890) .\end{array} \\
\end{array}$ \\
\hline & & \multirow{2}{*}{ Fidelidad en las cosas pequeñas } & La Educación, 108, 109 (1903). \\
\hline & & & Testimonies for the Church. 2:310 (1869). \\
\hline & & \multirow{3}{*}{ El esmero } & $\begin{array}{l}\text { The Youth's Instructor, } 19 \text { de febrero de } \\
1903\end{array}$ \\
\hline & & & Consejos para los Maestros, 61; 51 (1897). \\
\hline & & & La Educación, 108, 109 (1903). \\
\hline & & Los actos de la vida & Testimonies for the Church 4:657 (1881) \\
\hline & & Sujetar los pensamientos a Cristo & $\begin{array}{l}\text { Historia de los Patriarcas y Profetas, } 175 \\
\text { (1917). }\end{array}$ \\
\hline & & $\begin{array}{l}\text { No transigir con los defectos de } \\
\text { carácter }\end{array}$ & Consejos para los Maestros, 251 (1913). \\
\hline \multirow{7}{*}{3} & \multirow{7}{*}{$\begin{array}{l}\text { Relación con } \\
\text { Dios }\end{array}$} & \multirow{7}{*}{ Relación con Dios } & $\begin{array}{l}\text { White. E. G. (1911). The acts of the apostle. } \\
\text { 483. }\end{array}$ \\
\hline & & & The Review and Herald, June 12, 1888. \\
\hline & & & Testimonies for the Church 4:376 (1881) \\
\hline & & & $\begin{array}{l}\text { Palabras de Vida del Gran Maestro, 266; } \\
231 \text { (1900). }\end{array}$ \\
\hline & & & Obreros Evangélicos, 302, 303 (1915). \\
\hline & & & Testimonies for the Church 4:616 (1881). \\
\hline & & & $\begin{array}{l}\text { The Youth's Instructor, } 19 \text { de febrero de } \\
1903 .\end{array}$ \\
\hline
\end{tabular}




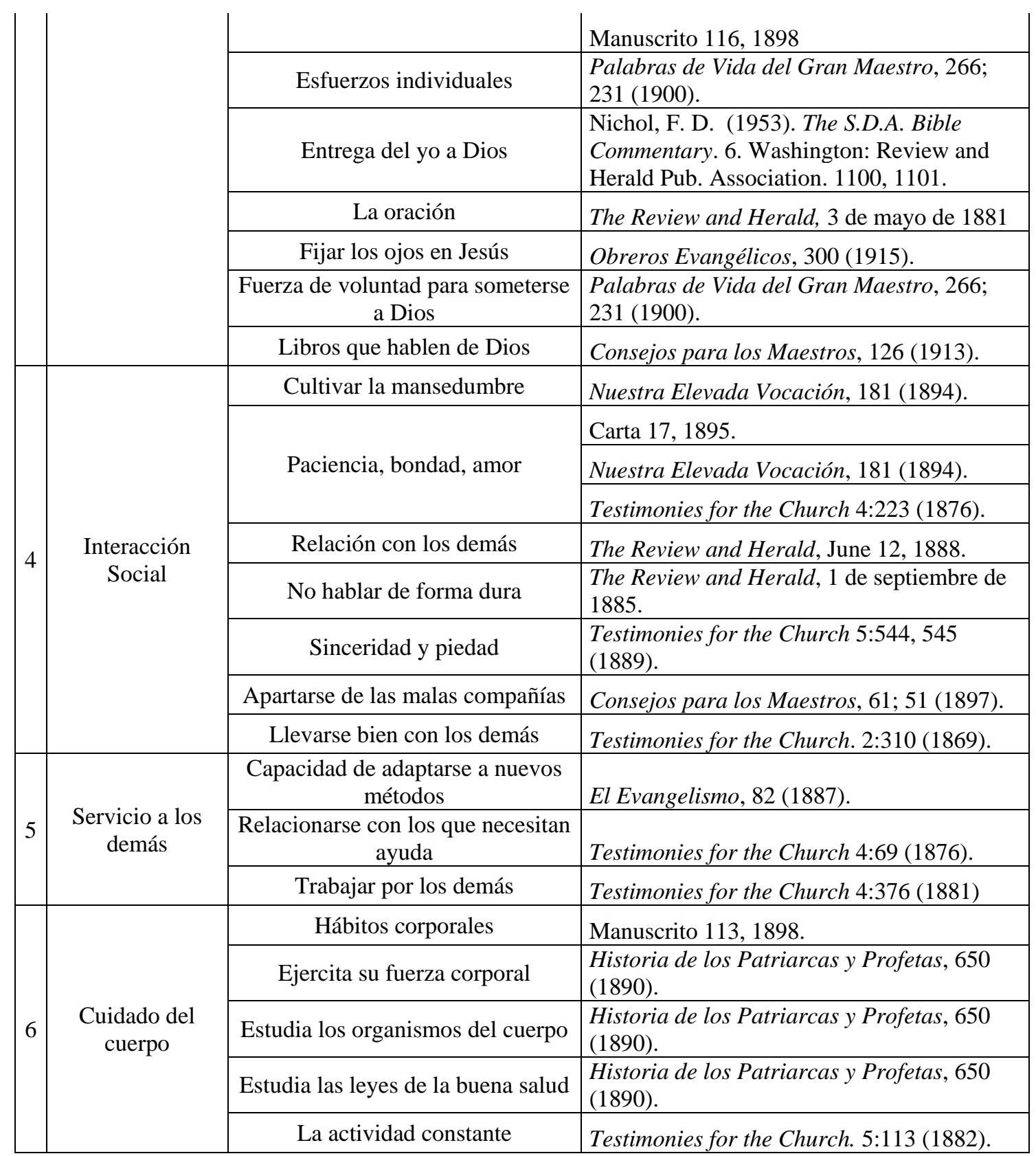

Se tomaron datos, a partir de fuentes determinadas, las cuales permitieron al presente estudio, llegar a las aulas para llevar a cabo una aproximación al currículo y su relación con la formación del carácter, esto se dio en la Universidad Peruana Unión, pues es un entorno educativo que pretende alcanzar estos objetivos.

A través del proceso de triangulación metodológica se analizaron los datos obtenidos de las fuentes con la finalidad de observar cómo se comportaba el concepto de la formación del carácter en una aproximación al currículo desde perspectivas diferentes. De esta manera se garantizó que lo encontrado se haya observado desde diferentes componentes, evitando así una opinión sesgada de lo hallado. 
Es así que se procedió a entrecruzar la información obtenida desde las siguientes fuentes: el análisis del documento curricular, el sílabo; la entrevista a un estudiante de cada materia (de formación general, de formación de especialidad y de nivel de posgrado), y observar la clase dictada para encontrar que tan próximos están los cursos de las categorías halladas en el estudio, demostrando un hilo conector a lo largo del mismo.

Gracias al organizador gráfico de información (OGI) se pudo sistematizar una gran cantidad de información presentada en texto de manera gráfica, respondiendo así el procesamiento de información, basado en esquemas mentales. Vemos la siguiente figura donde se destacan las categorías encontradas en los escritos de los primeros años del ministerio de Elena G. de White.

1. Manejo de los pensamientos

2. Relación con Dios

3. Servicio a los demás

4. Interacción Social 


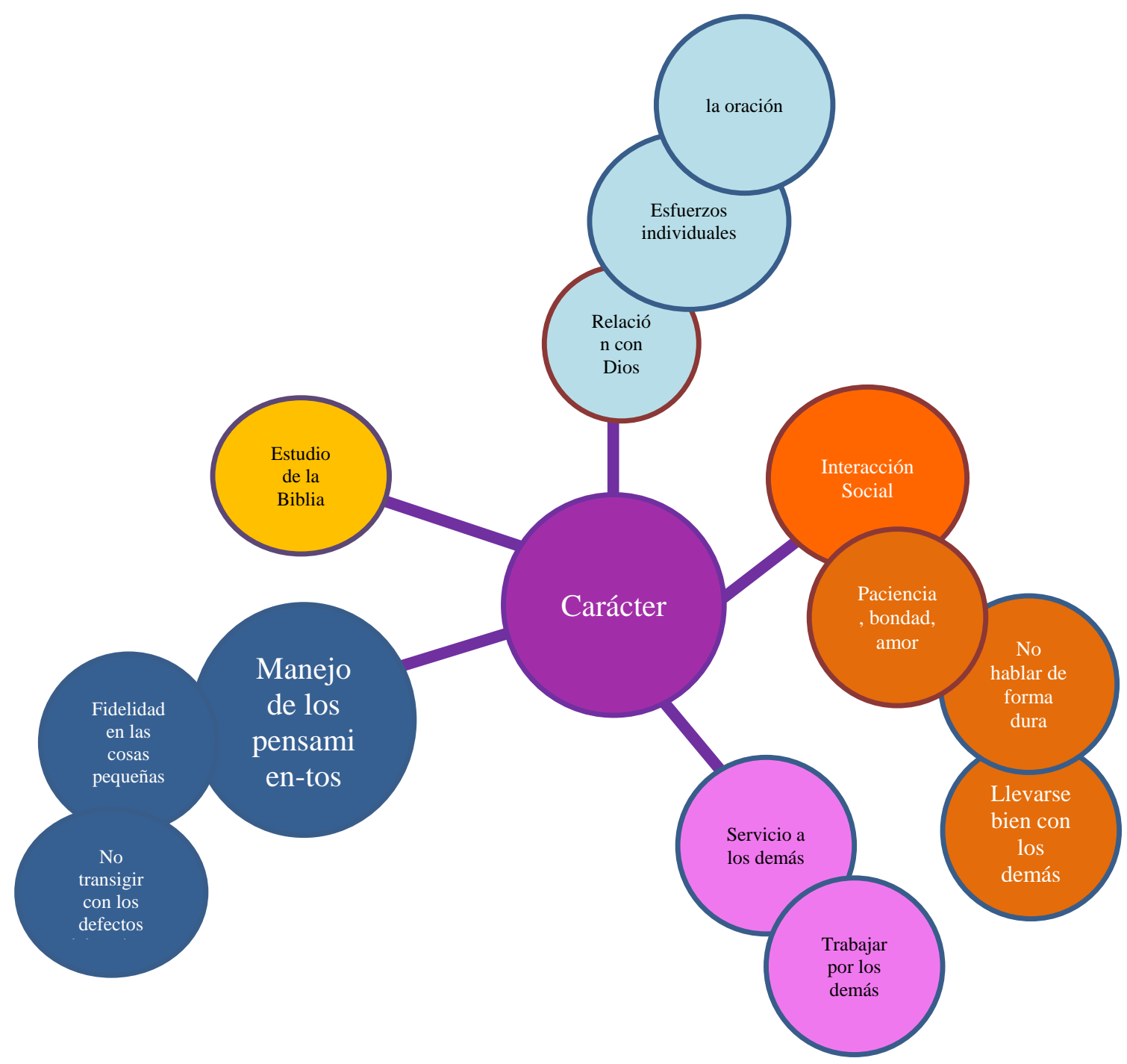

Figura 1. OGI "El pulpo" $1844-1885$

Teniendo en cuenta el evento histórico del congreso de Minneapolis de la Iglesia Adventista (IASD), encontramos una diferencia significativa entre las categorías emergentes en los primeros años del ministerio de Elena G. de White en relación al tema del carácter y los siguientes años después del congreso.

Se debe mencionar que el congreso de Minneapolis adquiere importancia por ser el punto central de referencia que marca la transición del pensamiento de la salvación por la ley hacia la salvación por la gracia permeando con este pensamiento, todas las creencias de la IASD, incluyendo la concepción de la formación del carácter.

En la siguiente figura se observa las categorías del carácter en todo el ministerio de Elena de White, viendo como de manera progresiva su pensamiento de carácter se hizo más completo y abarcante. 
1. Estudio de la Biblia

2. Manejo de pensamientos

3. Relación con Dios

4. Servicio a los demás

5. Interacción Social

6. Cuidado del cuerpo

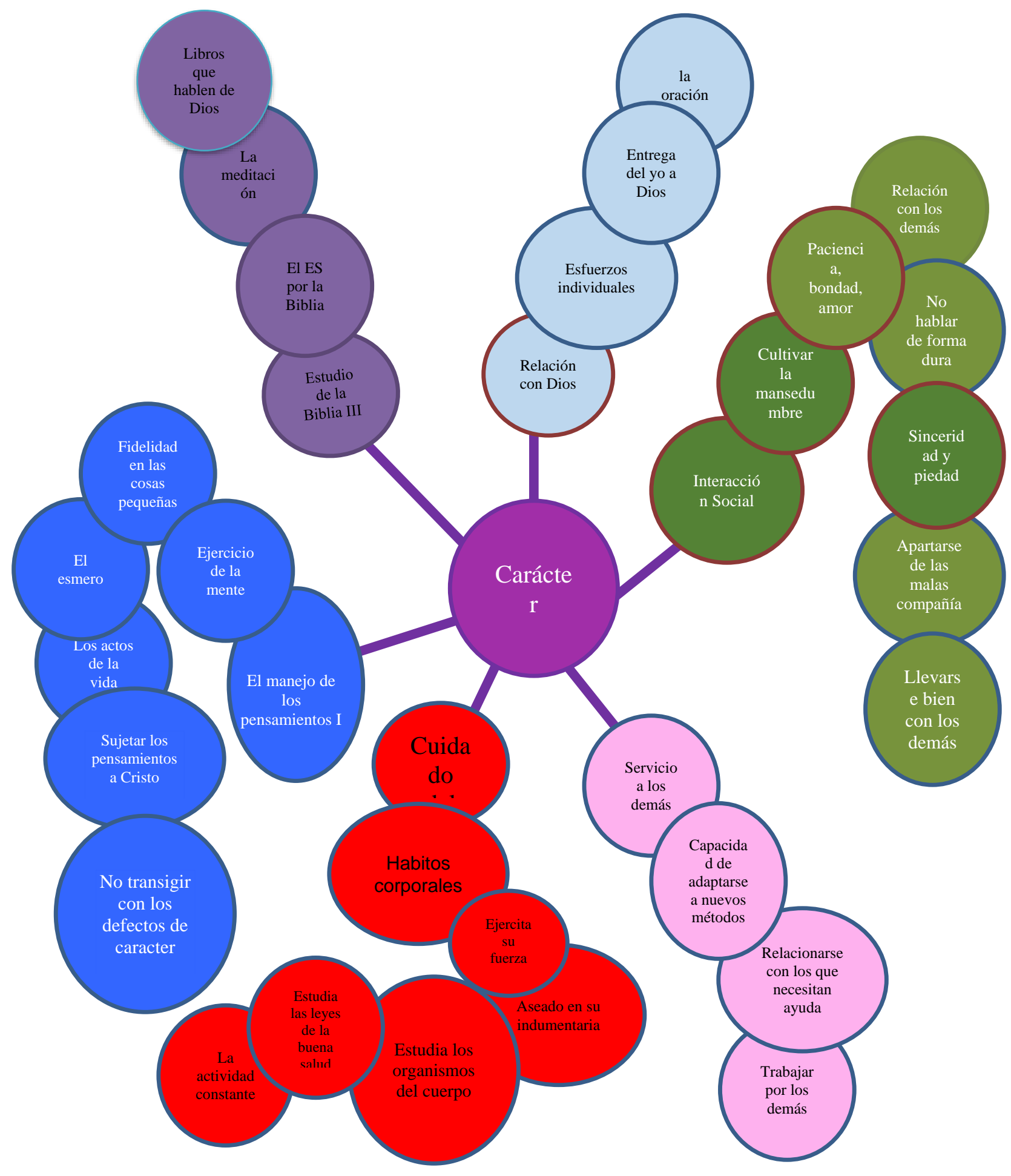


Figura 2. OGI "El pulpo" $1844-1915$

\section{Conclusiones}

Al finalizar esta investigación, se puede encontrar que la educación integral motiva a sumar toda competencia a favor de la formación del carácter.

La primera reflexión es sobre el enfoque cualitativo y sobre la complejidad de la natural y creciente transformación de una investigación cualitativa, siendo a la vez mi persona, el instrumento de investigación, y quien determinó sus propias fuentes para tomar datos de la realidad.

Se encuentra que en este enfoque se maximizan muchas competencias espirituales, cognitivas, sociales y personales, pues el camino a seguir al investigar, lo tiene la realidad misma y el investigador es una analítico quien aprecia, cuándo, cómo, para qué y por qué, se da la trasformación de la investigación, comparte como se va sistematizando, precisando y con esto arrojando valiosos hallazgos, haciendo viva y dinámica la experiencia investigativa.

En la historia, los avances en la formación del carácter, permiten encontrar que, involuntariamente algunas inconsistencias. Como refiere la situación encontrada a lo largo de estos periodos históricos, siendo que hasta 1888 no aparecen el estudio de la Biblia y el cuidado del cuerpo como categorías formadoras del carácter. Luego de 1888 aparecieron en abundancia.

La triangulación metodológica, demuestra una necesaria nivelación en los esfuerzos, para formar el carácter desde las aulas universitarias, siendo el 50\% débil en la presencia del estudio de la Biblia, la relación con Dios, y el cuidado del cuerpo; y, 50\% fuerte en las categorías del manejo de los pensamientos, el servicio a los demás y la interacción social (Martinez, 2017).

Se deben de tener en cuenta los hallazgos para crear un compromiso de los implicados y todas las dimensiones del currículo, en los diferentes sistemas educativos. Desde el nivel preescolar hasta el posgraduado. En necesario un compromiso de toda la comunidad educativa, pues todos ellos educan para formar el carácter.

\section{Recomendaciones}


La primera recomendación consiste en revisar las categorías propuestas en el presente estudio en cada una de las partes de la construcción de todo currículo que oriente el escenario educativo en cualquiera de sus niveles de formación.

La segunda recomendación gira en torno a una de las categorías denominada cuidado del cuerpo, la cual en la triangulación metodológica se presenta como las más débil, lo que nos lleva a recomendar el institucionalizar el cuidado del cuerpo tomando todas las medidas necesarias para ejecutar ello.

La tercera recomendación refiere el tener en cuenta las seis categorías encontradas en toda actividad técnico pedagógica; desde el nivel pre escolar, hasta el nivel de posgraduado.

La cuarta recomendación se direcciona hacia responder a la necesidad de evidenciar la presencia de las categorías en todo documento curricular.

En una quinta recomendación se plantea el alcanzar que todo aquel que participa del escenario educativo se constituya en parte de la comunidad formadora del carácter, motivando a que se comprometa con tal labor porque su presencia impacta a los estudiantes sea este individuo parte del personal no docente o forme parte de la plana administrativa.

Finalmente como sexta recomendación tanto el hogar la escuela y la iglesia son entornos vinculados a la formación del carácter en los que vemos el impacto, pro presencia o ausencia, de las categorías analizadas en la presente investigación, ,pues el hogar vive funciones de escuela e iglesia, por la educación constante y el desarrollo de la vida espiritual; la escuela vive las funciones d hogar e iglesia al construir la vida espiritual de sus educandos y presenta e $\mathrm{n}$ unidad a sus miembros; $\mathrm{y}$ finalmente la iglesia es en todas sus formas, un centro de enseñanza-aprendizaje, pues cumple la labor formadora de una escuela, así como también posee características de un hogar, manifestando lazos de afecto entre sus miembros, propios de una familia.

Cada uno de nosotros tiene una labor que hacer para el tiempo y la eternidad. Dios aborrece (la cursiva es nuestra) la indiferencia con respecto a la formación del carácter (Carta 223, 1903).

\section{Referencias}

Fowler, J. M., (1977). The Concept of Character Development in the Writings of Ellen

G. White. Dissertations. 374. Recuperado de http://digitalcommons.andrews.edu/dissertations/374

Grajales, T. (2005). El clima escolar y la formación del carácter cristiano. Recuperado de 
https://www.google.com/url?sa=t\&rct=j\&q=\&esrc=s\&source=web\&cd=10\&ved=0a hUKEwjsuOPQibXWAhXLGZAKHaXcA6IQFghiMAk\&url=http\%3A\%2F\%2Fojs. um.edu.mx\%2Findex.php\%2Fmemorias\%2Farticle\%2Fdownload\%2F85\%2F10\&us $\mathrm{g}=$ AFQjCNFbrhfH-T4fboE_03y7Whg_ZkgKUw

Hernández, S. R., Fernández, C. C., y Baptista, L. P. (2010). Metodología de la investigación. México, D.F: McGraw-Hill Education.

Mertens, D.M. (2005). Research and evaluation in education and psychology. Integrating diversity with quantitative, qualitative, and mixed methods (2 edition). Thousand Oaks: Sage.

Nichol, F. D. (1953). The S.D.A. Bible Commentary. 6. Washington: Review and Herald Pub. Association. 1100, 1101. (1898).

White, E. G. (1872). Carta 27

White, E. G. (1888). The Review and Herald, June 12.

White, E. G. (1967), Mensajes selectos. Mountain View, Calif: Publicaciones Interamericanas. 215, 317, 318,

White, E. G. (1971), Consejos para los maestros. Mountain View, Calif: Pacific Press Pub. Association. 85, 97, 104.

White, E. G. (1971). Joyas de los testimonios: consejos para la iglesia: seleccionados de los testimonios, t. 1. Mountain View, Calif: Pacific Press Pub. Association. 16.

White, E. G. (1973). La maravillosa gracia de Dios. Buenos Aires: Asociación Casa Editora Sudamericana. 55

White, E. G. (1981), Notas biográficas de Elena G. de White: narración autobiográfica hasta 1881 y resumen de su vida posterior basado en fuentes originales. Mountain View, Calif: Publicaciones Interamericanas, 182, 183.

White, E. G. (1988). Exaltad a Jesús: Lecturas devocionales para cada día del año. Mountain View, Calif: Pacific Press Pub. Association.

White, E. G. (1998). Mente carácter y personalidad. Mountain View, Calif: Pacific Press Pub. Association. 75.

White, E. G. (2000). El camino a Cristo. Colmenar Viejo. Madrid: Safeliz. 99.

White, E. G. (2009). La educación. Doral, Florida: Asociación Publicadora Interamericana. 108, 109.

White. E. G. (1911). The acts of the apostle. 483

White. E. G. (1869). Testimonies for the Church. 2:310

White. E. G. (1869). Testimonies for the Church. 2:310

White. E. G. (1876). Testimonies for the Church 4:223

White. E. G. (1876). Testimonies for the Church 4:69

White. E. G. (1876). Testimonies for the Church 4:69

White. E. G. (1881). Testimonies for the Church 4:376

White. E. G. (1881). Testimonies for the Church 4:376

White. E. G. (1881). Testimonies for the Church 4:376 
White. E. G. (1881). Testimonies for the Church 4:616

White. E. G. (1881). Testimonies for the Church 4:657

White. E. G. (1882). Testimonies for the Church. 5:113

White. E. G. (1882). Testimonies for the Church. 5:113

White. E. G. (1887). El Evangelismo, 82

White. E. G. (1889). Testimonies for the Church 5:544, 545

White. E. G. (1890). Historia de los Patriarcas y Profetas, 650

White. E. G. (1890). Historia de los Patriarcas y Profetas, 650

White. E. G. (1894). Nuestra Elevada Vocación, 181

White. E. G. (1894). Nuestra Elevada Vocación, 181

White. E. G. (1895). Carta 17.

White. E. G. (1897). Consejos para los Maestros, 61; 51

White. E. G. (1897). Consejos para los Maestros, 61; 51

White. E. G. (1898). Manuscrito 113.

White. E. G. (1898). Manuscrito 116.

White. E. G. (1900). Palabras de Vida del Gran Maestro, 266; 231

White. E. G. (1900). Palabras de Vida del Gran Maestro, 266; 231

White. E. G. (1903). La Educación, 108, 109

White. E. G. (1905). El Ministerio de Curación, 364

White. E. G. (1911). The acts of the apostle. 483.

White. E. G. (1913). Consejos para los Maestros, 126

White. E. G. (1913). Consejos para los Maestros, 251

White. E. G. (1915). Obreros Evangélicos, 300

White. E. G. (1915). Obreros Evangélicos, 302, 303

White. E. G. (1917). Historia de los Patriarcas y Profetas, 175

White. E. G. The Review and Herald, 1 de septiembre de 1885.

White. E. G. The Review and Herald, 25 de julio de 1899.

White. E. G. The Review and Herald, 3 de mayo de 1881

White. E. G. The Review and Herald, 3 de mayo de 1881

White. E. G. The Review and Herald, June 12, 1888.

White. E. G. The Review and Herald, June 12, 1888.

White. E. G. The Review and Herald, June 12, 1888.

White. E. G. The Youth's Instructor, 19 de febrero de 1903

White. E. G. The Youth's Instructor, 19 de febrero de 1903. 
Zavala, F. (2006). Control de calidad para tu mente: Guía para excelencia mental y espiritual. Colmenar Viejo, Madrid: Editorial Safeliz. 45, 109. 